\title{
Dietary emulsifiers directly alter human microbiota composition and gene expression ex vivo potentiating intestinal inflammation
}

\author{
Benoit Chassaing, ${ }^{1}$ Tom Van de Wiele, ${ }^{2}$ Jana De Bodt, ${ }^{2}$ Massimo Marzorati, ${ }^{2}$ \\ Andrew T Gewirtz ${ }^{1}$
}

- Additional material is published online only. To view (http://dx.doi.org/10.1136/ gutjnl-2016-313099).

${ }^{1}$ Center for Inflammation, Immunity and Infection, Institute for Biomedical Sciences, Georgia State University, Atlanta, Georgia, USA

${ }^{2}$ Center of Microbial Ecology and Technology (CMET), Faculty of Bioscience Engineering, Ghent University, Ghent Belgium

\section{Correspondence to} Dr Benoit Chassaing, Center for Inflammation, Immunity, and Infection, Institute for Biomedical Sciences, Georgia State University, Atlanta, GA 30312, USA;

bchassaing@gsu.edu and Pr Tom van de Wiele, Center of Microbial Ecology and Technology (CMET), Faculty of Bioscience Engineering, Ghent University, Ghent, Belgium

Received 23 September 2016 Revised 13 February 2017 Accepted 28 February 2017 Published Online First 21 March 2017 please visit the journal online

ABSTRACT

Objective The intestinal microbiota plays a central role in the development of many chronic inflammatory diseases including IBD and metabolic syndrome. Administration of substances that alter microbiota composition, including the synthetic dietary emulsifiers polysorbate 80 (P80) and carboxymethylcellulose (CMC), can promote such inflammatory disorders. However, that inflammation itself impacts microbiota composition has obfuscated defining the extent to which these compounds or other substances act directly upon the microbiota versus acting on host parameters that promote inflammation, which subsequently reshapes the microbiota.

Design We examined the direct impact of CMC and P80 on the microbiota using the mucosal simulator of the human intestinal microbial ecosystem (M-SHIME) model that maintains a complex stable human microbiota in the absence of a live host.

Results This approach revealed that both P80 and CMC acted directly upon human microbiota to increase its proinflammatory potential, as revealed by increased levels of bioactive flagellin. The CMC-induced increase in flagellin was rapid (1 day) and driven by altered microbiota gene expression. In contrast, the P80-induced flagellin increase occurred more slowly and was closely associated with altered species composition. Transfer of both emulsifier-treated M-SHIME microbiotas to germfree recipient mice recapitulated many of the host and microbial alterations observed in mice directly treated with emulsifiers.

Conclusions These results demonstrate a novel paradigm of deconstructing host-microbiota interactions and indicate that the microbiota can be directly impacted by these commonly used food additives, in a manner that subsequently drives intestinal inflammation.

\section{INTRODUCTION}

A panel of chronic inflammatory diseases is associated with alterations in the species and/or CrossMark

To cite: Chassaing B, Van de Wiele T, De Bodt J, et al. Gut 2017;66:1414-1427. genomic composition of the intestinal microbiota. In animal models, such diseases are typically ameliorated in the absence of a microbiota, achieved via use of antibiotics or germ-free (GF) methodologies, indicating that the presence of a microbiota is possibly linked to disease development. Moreover,

\section{Significance of this study}

What is already known on this subject?

- Chronic inflammatory diseases are associated with alterations in the species and genomic composition of the intestinal microbiota.

- Dietary emulsifiers polysorbate 80 (P80) and carboxymethylcellulose (CMC) consumption results in microbiota encroachment into the mucus, alterations in microbiota composition and development of chronic inflammation.

- Inflammation itself can impact microbiota composition, making it very difficult to discern the extent to which these compounds act directly upon the microbiota versus promoting host inflammation, which subsequently reshapes the microbiota.

What are the new findings?

- CMC and P80 do not impact the intestinal microbiota nor host in mice harbouring a pathobiont-free microbiota.

- Use of the mucosal simulator of the human intestinal microbial ecosystem (M-SHIME) reveals that both P80 and CMC directly alter the microbiota, increasing its proinflammatory potential.

- When transplanted to germ-free mice, CMC-treated and P80-treated M-SHIME suspensions promote low-grade inflammation-associated phenotypes indicating that the direct effects of these emulsifiers on the microbiota are sufficient to drive low-grade inflammation and metabolic disease.

How might it impact on clinical practice in the foreseeable future?

- The observation that emulsifiers CMC and P80 directly impact human microbiomes in a manner reminiscent of their effects in mice supports the use of this model as a practical means to test food additives for safety.

- Our results demonstrate the utility of the M-SHIME system as a tool to explore underlying mechanisms of any host/microbiota alterations. 
the observation that transfer of microbiota from diseased hosts-either mice or humans-to GF mice can transfer some features of disease suggests that disease-associated alterations in microbiota composition are not purely a consequence of diseases, but, rather, play a role in driving disease development. On the other hand, inflammation itself can lead to potent alterations in microbiota composition and does so in a manner that displays similar features that are observed in some inflammatory diseases, for example, loss of $\alpha$-diversity and/or increase in $\gamma$-proteobacteria. ${ }^{1-7}$ Together, these results suggest that alterations in gut microbiota that have been associated with inflammatory diseases are likely both a cause and consequence of inflammation. In this respect, it may be very difficult to determine the extent to which a given substance-whose administration results in altered microbiota composition and gut inflammation-acted directly upon the microbiota and/or the host. An example of this concept can be seen in our recent work on the food additives carboxymethylcellulose (CMC) and polysorbate 80 (P80). ${ }^{8}$ CMC and P80 are commonly used synthetic emulsifiers that are added to a variety of processed foods to enhance texture and extend shelf-life. Based on the epidemiology of their usage, and the notion that their detergent properties might promote bacterial translocation across mucosal surfaces, these emulsifiers are hypothesised to have contributed to increased incidence of chronic gut inflammatory diseases. ${ }^{8-11}$ In support of this hypothesis, we observed that administration of CMC and P80 to mice resulted in microbiota encroachment into the mucus, alterations in microbiota composition, including an increase of bacteria that produced proinflammatory flagellin (FliC) and lipopolysaccharide (LPS), and development of chronic inflammation. ${ }^{8} 12$ Such inflammation was associated with low-grade inflammation and metabolic disease in wild-type (WT) mice and increased incidence/severity of overt colitis in interleukin-10 (IL-10) $)^{-/-}$mice, which are prone to this disorder. We hypothesised that such events might be initiated by these emulsifiers directly interacting with the mucus, thereby promoting bacterial encroachment that would activate the innate immune system to induce antimicrobial gene expression, thereby more broadly changing microbiota composition. In accordance with this possibility, both CMC and P80 administration resulted in a thinner mucus layer. However, such emulsifierinduced mucus thinning was not observed in GF mice, nor did we observe an altered localisation of fluorescent beads-of the same size of bacteria-in GF animals in presence of CMC or P80. ${ }^{8}$ Such findings argued against the notion that these emulsifiers were directly impacting the mucus layer and, rather, suggest the possibility that CMC and P80 might directly impact the intestinal microbiota.

Hence, the central goal of this study was to investigate the extent to which CMC and P80 might directly alter microbiota using a mucosal simulator of the human intestinal microbial ecosystem (M-SHIME) model, which allows a complex human microbiota to be stably maintained in vitro in the absence of host cells. ${ }^{13-16}$ We observed that P80 and CMC altered the microbiota in the M-SHIME model in a manner reminiscent of, and capable of recapitulating, their effects previously observed in mice, thus suggesting that the microbiota may be a key direct target of CMC and P80.

\section{METHODS}

Mice

$\mathrm{C} 57 \mathrm{BL} / 6$ mice were maintained in gnotobiotic conditions in a GF or altered Schaedler flora (ASF) state in a Park Bioservices isolator as previously described. ${ }^{8}$ C57BL/6 $\mathrm{Rag}^{-/-}$ (recombination activating gene) mice were maintained in specific pathogen-free housing. All mice were bred and housed at Georgia State University (Atlanta, Georgia, USA) under institutionally approved protocols (IACUC \# A14033). Mice were fed autoclaved LabDiet Rodent Chow \# 5021 or irradiated (20$40 \mathrm{kGy}$ ) purified high-fat diet (HFD) D12492-1.5V from Research Diet (New Brunswick, New Jersey, USA) (see online supplementary table S1). Experiments used both male and female mice (comparisons within a gender) except for figures 7 and 8 that used female mice. ASF mice were established by colonising WT C57BL/6 GF mice with the complete ASF (eight strains) using faeces purchased from ASF Taconic (Hudson, New York, USA) and resuspended in drinking water.

\section{Fasting blood glucose measurement}

Mice were placed in a clean cage and fasted for 5 hours or 15 hours. Blood glucose concentration was then determined using a Nova Max Plus Glucose Meter and expressed in mg/dL.

\section{Emulsifier treatment of the M-SHIME system}

The direct effect of emulsifier on intestinal microbiota was studied in an M-SHIME system, a dynamic in vitro model which simulates the lumen-associated and mucus-associated human intestinal microbial ecosystem (ProDigest-Ghent University, Ghent, Belgium). ${ }^{13-15}$ This model consists of consecutive pH-controlled, stirred (200 rpm), airtight, double-jacketed glass vessels kept at $37^{\circ} \mathrm{C}$ and under anaerobic conditions by daily flushing with $\mathrm{N}_{2}(15 \mathrm{~min})$. The set-up used in this study consisted of a stomach and a small intestine vessel and eight (experiment represented figures 3-5, 7-9 and online supplementary figures S1-S13) or nine (experiment represented figure 6) proximal colon vessels in parallel. The system was operated, and simulation media were prepared as described earlier. ${ }^{13-15}$ The colon vessels were inoculated at the start with $40 \mathrm{~mL}$ human faecal suspension in $500 \mathrm{~mL}$ sterile nutritional medium (per L: $3 \mathrm{~g}$ yeast extract, $1 \mathrm{~g}$ special peptone (Oxoid, Aalst, Belgium), $4 \mathrm{~g}$ commercial porcine gastric mucin (Sigma-Aldrich, Bornem, Belgium) and $0.5 \mathrm{~g} \mathrm{~L}$-cysteine). After an initial static incubation of 18 hours, $140 \mathrm{~mL}$ sterile nutritional medium at $\mathrm{pH} 2$ per colon vessel was supplemented to the stomach vessel: this procedure was conducted three times a day. Stomach digest suspension was pumped into the small intestine vessel together with $60 \mathrm{~mL}$ bile and pancreatic juice (per L: $12.5 \mathrm{~g} \mathrm{NaHCO}_{3}, 6 \mathrm{~g}$ dehydrated bile extract (Oxgall, Difco) and $0.9 \mathrm{~g}$ pancreatin (Sigma)) per colon vessel. Small intestinal digest suspension was distributed over the different proximal colon vessels, which contained simulated colon microbiota. To simulate colon conditions, the residence time in the colon vessels was 20 hours and $\mathrm{pH}$ was controlled at 6.15-6.40, which are both in the range of in vivo observations. ${ }^{17}{ }^{18}$ Mucus microbiota were simulated by adding carriers coated with agar containing commercially available porcine gastric mucin (Sigma-Aldrich). ${ }^{13}$ Sixty mucin agar-covered microcosms in a polyethylene netting were added to each colon vessel to obtain an appropriate mucus surface area. Every 2 days, two-thirds of the mucin agar-covered microcosms were replaced by fresh sterile ones under a flow of $\mathrm{N}_{2}$ to prevent disruption of anaerobic conditions. Seven days after inoculation, colon vessels were treated with either CMC or P80 at a final concentration of $1.00 \%, 0.50 \%, 0.25 \%$ or $0.10 \%$. Lumen and mucin agar samples were taken every 2 days. Mucin agar-covered microcosms were washed with sterile phosphate-buffered saline (PBS) to remove lumen bacteria. Mucin agar was removed from 
microcosms, homogenised and stored immediately at $-20^{\circ} \mathrm{C}$ until further analysis.

\section{M-SHIME metatranscriptomic analysis}

Total RNAs were extracted from M-SHIME suspension using Qiagen RNeasy kit, according to the manufacturer's protocol. After purification, RNA concentration and integrity were determined using Epoch Microplate Spectrophotometer (Bio-Tek, Winooski, Vermont, USA) and agarose gel electrophoresis, respectively. Total RNA was then prepared for sequencing using Illumina TruSeq RNA kit and according to the manufacturer's protocol. Briefly, rRNA were depleted using Illumina Ribo-Zero magnetic kit according to the manufacturer's protocol. rRNA-depleted RNA were fragmented and converted to cDNA. After end repair and ligation of adapters, mRNA libraries were amplified by PCR and validated using BioAnalyser, according to manufacturer's recommendations. The purified library was then subjected to sequencing using an Illumina NextSeq500 at the Cornell University sequencing core (Ithaca, New York, USA). Sequencing data obtained were analysed through MG-RAST pipeline, ${ }^{19}$ and metatranscriptomes were determined and summarised using Kyoto Encyclopedia of Genes and Genomes (KEGG) orthology (KO), ${ }^{20}{ }^{21}$ Clusters of Orthologous Groups of proteins $^{22}$ and non-supervised orthologous groups ${ }^{23}$ for each sample and compared using either principal coordinate analysis of the Euclidean distance, volcano plot created using $\mathrm{R}$ software, heatmap representation using Gene-E of pathways of interest. ${ }^{24}$ Unprocessed sequencing data are deposited at MG-RAST http:// metagenomics.anl.gov/linkin.cgi? project $=\operatorname{mgp} 16059$, samples mgs411584, mgs411587, mgs411590, mgs411593, mgs411596, mgs411599, mgs411602, mgs411605, mgs411608, mgs411611, mgs411614, mgs411617, mgs411620, mgs411623, mgs411626, mgs411629, mgs411632, mgs411635, mgs411638, mgs411641, mgs411644, mgs411647, mgs411650 and mgs411653.

\section{Microbiota transplantation of emulsifier-treated M-SHIME suspension}

GF C57BL/6 mice (3-4 weeks old for figures 8 and 9, 5-10 weeks old for online supplementary figure S13) were removed from isolator and were orally administered with $200 \mu \mathrm{L}$ of luminal M-SHIME suspension (day 11 sample). Transplanted mice were then housed in isolated ventilated cages, Isocages (Techniplast, West Chester, Pennsylvania, USA), ${ }^{25}$ and fed autoclaved Purina Rodent Chow \# 5021 or irradiated (20$40 \mathrm{kGy}$ ) purified HFD D12492-1.5V from Research Diet. For each condition (water-treated M-SHIME, CMC-treated M-SHIME, P80-treated M-SHIME), two cages containing one to three animals were used, with each cage receiving suspension from an independent M-SHIME vessel (for each condition, $\mathrm{N}=2$ vessels, each one transferred to a cage of one to three animals, for a total of three to five animals). Body weights were measured every week and are expressed as compared with the initial body weight (day 0) defined as 100\%. Fresh faeces were collected every other week for downstream analysis. Twelve (see online supplementary figure S13) or thirteen (figures 7-9 and online supplementary figures S9-S12) weeks post transplant, mice were fasted for 5-hours at which time blood was collected by retrobulbar intraorbital capillary plexus. Haemolysis-free serum was generated by centrifugation of blood using serum separator tubes (Becton Dickinson, Franklin Lakes, New Jersey, USA). Mice were then euthanised, and colon length, colon weight, spleen weight and adipose weight were measured. Organs were collected for downstream analysis.

\section{Statistical analysis}

Normal distribution of the data was assessed using D'Agostino-Pearson omnibus test. Significance was determined using t-test or two-way group analysis of variance (ANOVA) with Bonferroni's multiple comparisons test (GraphPad Prism software, V.6.01). Differences were noted as significant $* \mathrm{p} \leq 0.05$ for t-test and $\# \mathrm{p} \leq 0.05$ for two-way ANOVA. For clustering analysis on principal coordinate plots, categories were compared, and statistical significance of clustering was determined via Permanova.

\section{RESULTS \\ CMC and P80 do not impact intestinal microbiota or host in ASF mice}

In order to investigate the extent to which CMC and P80 might impact the host and/or microbiota in mice with a limited defined pathobiont-free microbiota, we administered CMC and P80 to mice colonised exclusively with ASF. As shown in figures 1 and 2, neither CMC nor P80 had a significant impact on a range of host or microbial parameters in ASF mice. Briefly, whether assessed globally by principal coordinate analysis or via comparison of individual species (Table 1), assay of relative levels of the eight ASF components by q-PCR indicated that neither CMC nor P80 impacted microbiota composition of ASF mice, nor did they alter faecal levels of Toll-like receptor (TLR)-agonists LPS and flagellin (figures 1 and 2). Moreover, in ASF mice, CMC and P80 administration did not result in microbiota encroachment (figure $2 \mathrm{~L}$, $\mathrm{M})$, mucus thinning or features of low-grade inflammation/metabolic syndrome that are induced by these compounds in mice with a complex microbiota. ${ }^{8}$ Together, these results argue against the notion that these emulsifiers have a strong direct effect on the host and rather suggest the possibility that more subtle effects on a complex microbiota may be proximal mediators of the effects of these compounds.

\section{P80 alters microbiota composition in the M-SHIME model}

Having not observed an impact of CMC and P80 on a host lacking a complex microbiota, we next used the converse approach of examining how these compounds impacted a complex microbiota in the absence of a host by using the M-SHIME model. Application of $16 \mathrm{~S}$ sequencing to luminal M-SHIME samples indicated that the bacterial composition of this model changed rapidly following initial inoculation with human faeces, but had largely stabilised 7 days post inoculation (see online supplementary figure S1A). Such stabilisation was associated with an approximate 50\% decline in $\alpha$-diversity, but nonetheless still afforded a stable complex microbiota for further study (see online supplementary figure S1B). Hence, we defined this point as time zero and subsequently added CMC, P80 or water (control) to multiple M-SHIME vessels maintained in parallel. As expected, when assayed prior to addition of emulsifiers, M-SHIMEs did not differ significantly from each other (figure 3A, B). However, in response to P80, microbiota composition of M-SHIMEs gradually shifted, such that by day 11, all P80-treated M-SHIMEs clearly clustered together quite distinctly from the other M-SHIME vessels (figure 3C). In contrast, the bacterial compositions of CMC-treated and control M-SHIMEs did not differ significantly from each other at any time point assayed (figure $3 \mathrm{~A}-\mathrm{C}$ ). In accordance with our visual assessment of the principal coordinates analysis (PCoA) plots, the UniFrac distances between control and P80-treated M-SHIMEs were significantly greater than those of any following (11 days) but not preceding ( -6 days) treatment (figure 3D and E). Such altered composition resulting from P80 treatment 

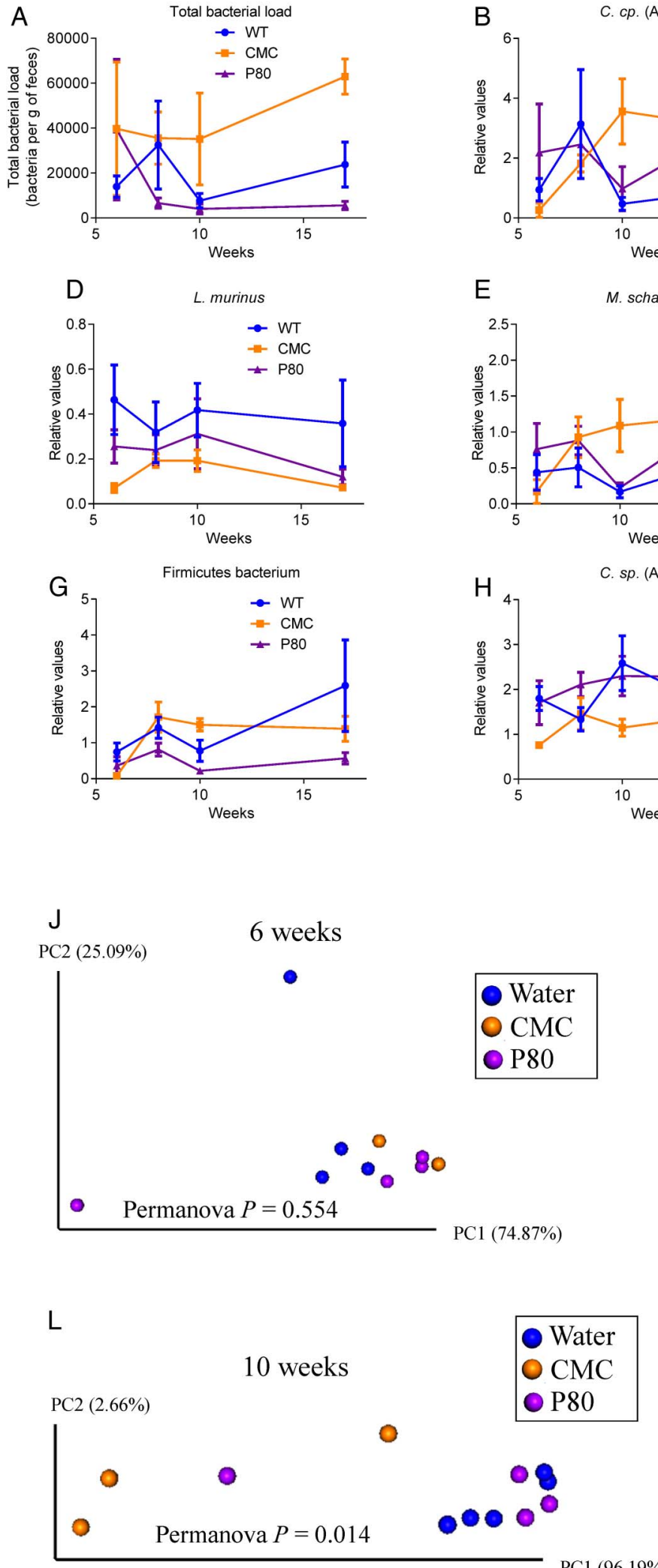
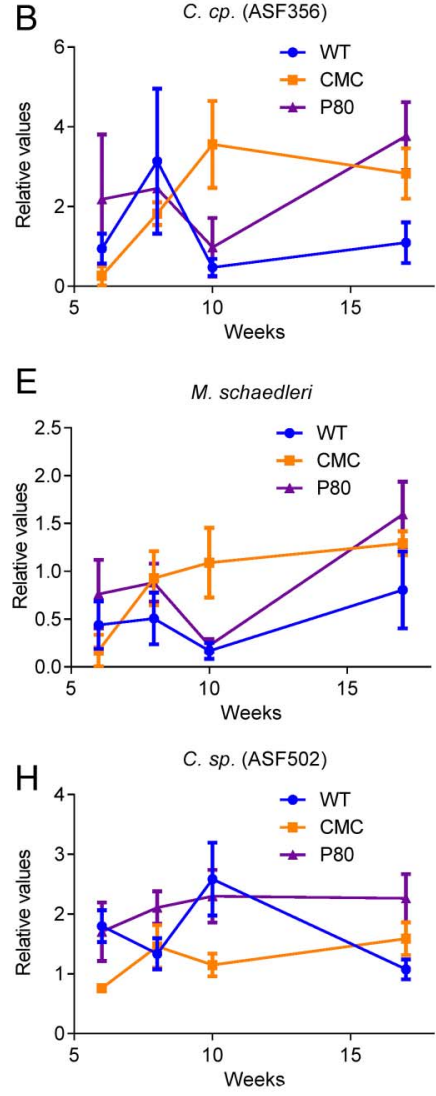

K PC2 (41.51\%)

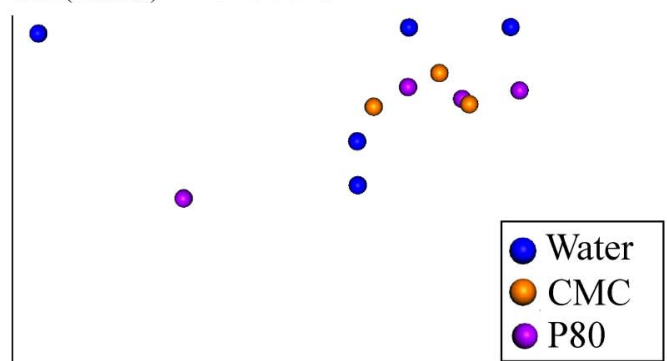

Permanova $P=0.936$

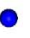

$\operatorname{PCl}(58.34 \%)$

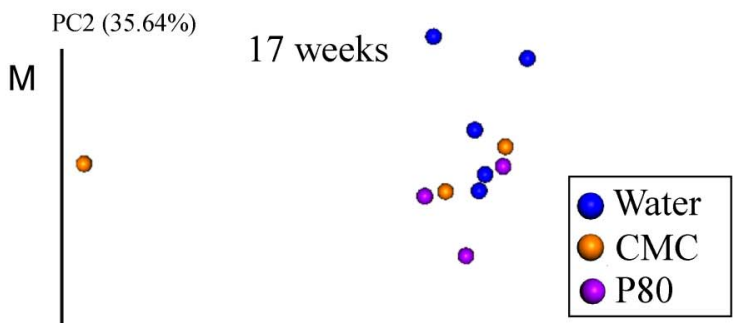

Permanova $P=0.033$

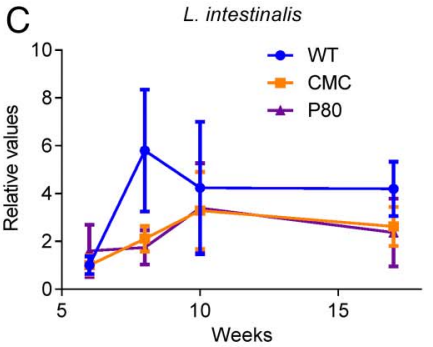

F
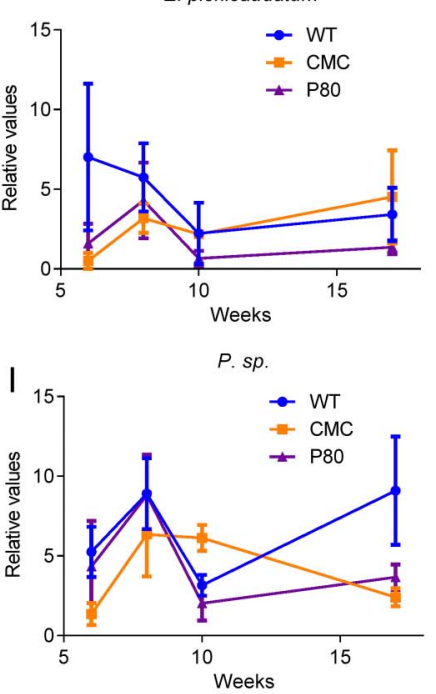

8 weeks

Figure 1 Carboxymethylcellulose (CMC) and P80 do not impact intestinal microbiota composition in altered Schaedler flora (ASF) mice. Six-week-old ASF C57BL/6 mice were exposed to CMC or P80 diluted in drinking water (1.0\%) for 11 weeks. (A-I) Total faecal DNA was extracted and subjected to q-PCR using specific primers for $16 \mathrm{~S}$ rRNA (A), Clostridium sp. (ASF 356) (B), Lactobacillus intestinalis (C), Lactobacillus murinus (D), Mucispirillum schaedleri (E), Eubacterium plexicaudatum (F), Firmicutes bacterium (G), Clostridium sp. (ASF 502) (H) and Parabacteroides sp. (I). Results are expressed as bacteria number per $\mathrm{mg}$ of stool (A), using a standard curve, or as relative values (B-I). (J-M) Principal coordinate analysis was performed using Euclidean distance with the eight ASF members at week $6(\mathrm{~J})$, week $8(\mathrm{~K})$, week $10(\mathrm{~L})$ and week $17(\mathrm{M})$. Data are the means $\pm \mathrm{SEM}(\mathrm{N}=3-6)$. For cluster analysis on principal coordinate plots, categories were compared and statistical significance of clustering were determined using Permanova method. 

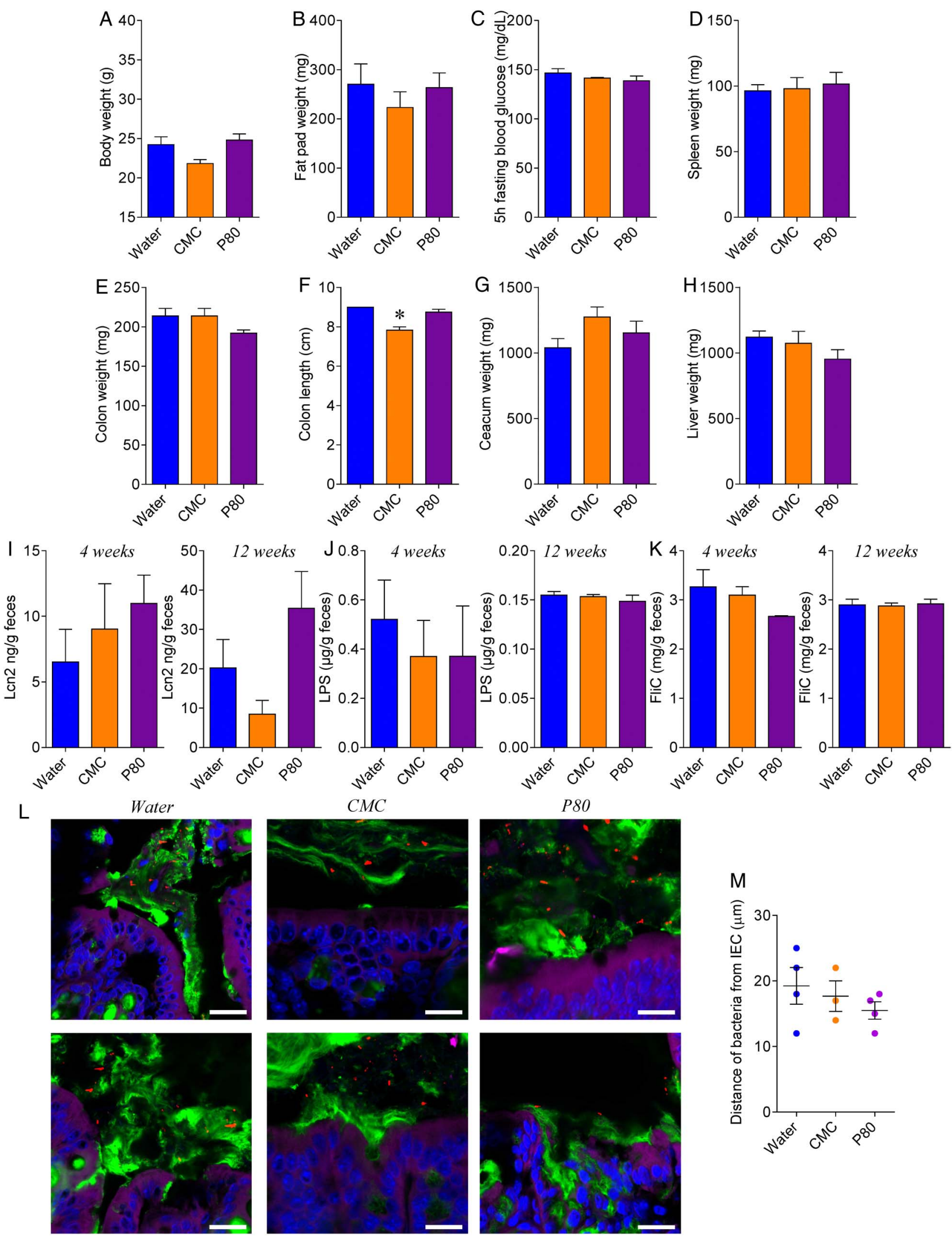

Figure 2 Carboxymethylcellulose (CMC) and P80 do not induce intestinal inflammation nor metabolic syndrome nor microbiota encroachment in altered Schaedler flora (ASF) mice. Six-week-old ASF C57BL/6 mice were exposed to CMC or P80 diluted in drinking water (1.0\%) for 11 weeks. (A) Final body weight, (B) fat pad weight, (C) 5 hours fasting blood glucose concentration, (D) spleen weight, (E) colon weight, (F) colon length, (G) caecum weight, (H) liver weight, (I) faecal Lcn2, (J) lipopolysaccharide (LPS) and (K) Flagellin (FliC) levels at 4 and 12 weeks. (L) Confocal microscopy analysis of microbiota localisation; Muc2 (green), actin (purple), bacteria (red) and DNA (blue). Bar=20 $\mu \mathrm{m}$. (M) Distances of the closest bacteria to intestinal epithelial cells (IEC) per condition over three high-powered fields per mouse. Data are the means $\pm \mathrm{SEM}(\mathrm{N}=3-6)$. Each treatment group was compared with the control group (water-treated), and statistical significance was determined using Student's t-test. 


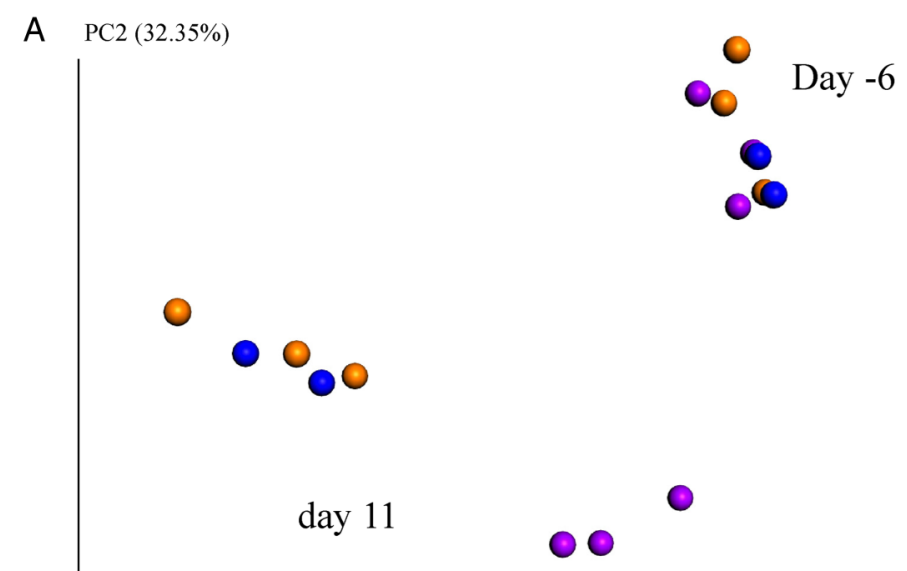

\section{- Water \\ - CMC \\ - P80}

PC1 (49.55\%)
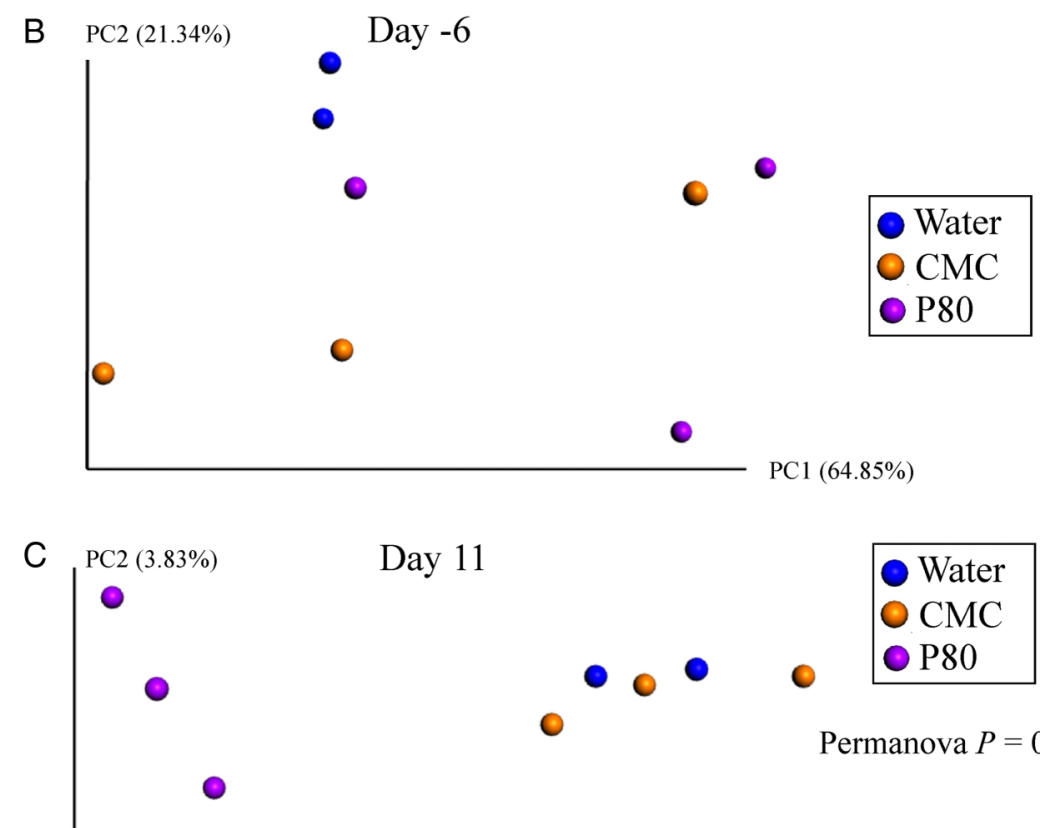

Day 11

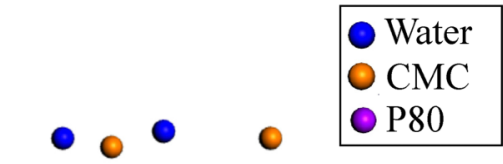

○

Permanova $P=0.042$
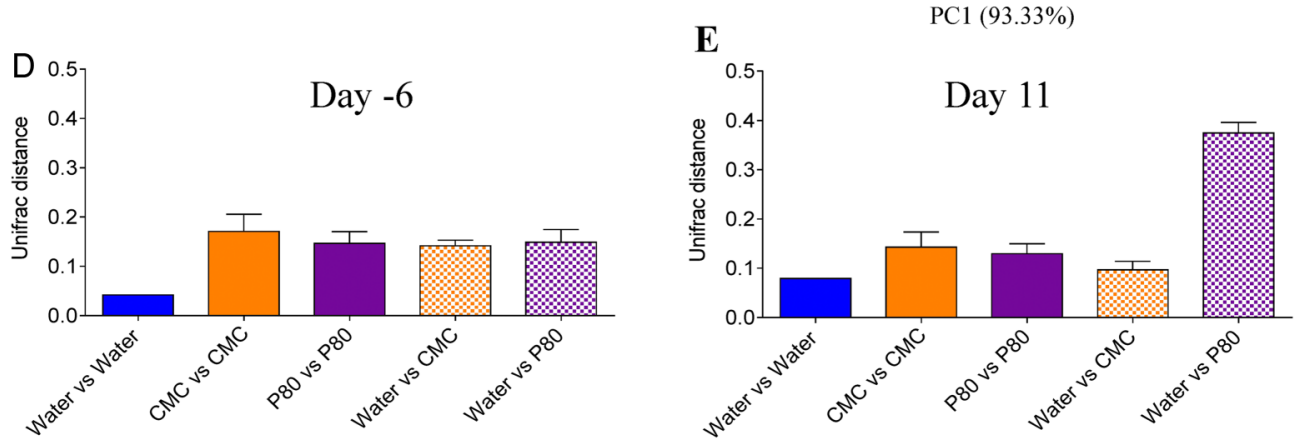

Figure 3 P80 alters mucosal simulator of the human intestinal microbial ecosystem (M-SHIME) microbiota composition. An eight-vessel M-SHIME system was set up and inoculated with fresh human faeces at day -7 . Following a 7-day stabilisation period, vessels were treated with either water $(\mathrm{N}=2)$, carboxymethylcellulose (CMC) $1 \%(\mathrm{w} / \mathrm{v}, \mathrm{N}=3)$ or $\mathrm{P} 801 \%(\mathrm{v} / \mathrm{v}, \mathrm{N}=3)$ for 13 days. Luminal microbiota composition was analysed using Illumina sequencing of the V4 region of 16S rRNA genes. (A-C) Principal coordinate analysis of the weighted UniFrac distance matrix at days -6 and $11(A)$, day -6 (B) and day 11 (C). (D and E) Average of the weighted UniFrac distance within group (water, CMC and P80) and between group (water vs CMC and water vs P80) has been calculated at days -6 (D) and 11 (E). Data are the means $\pm S E M(N=2-3)$. For cluster analysis on principal coordinate plots, categories were compared, and statistical significance of clustering was determined using the Permanova method. 
A

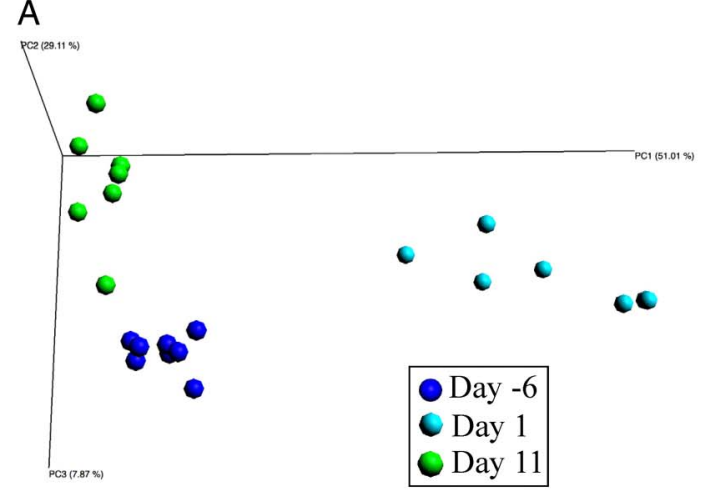

B

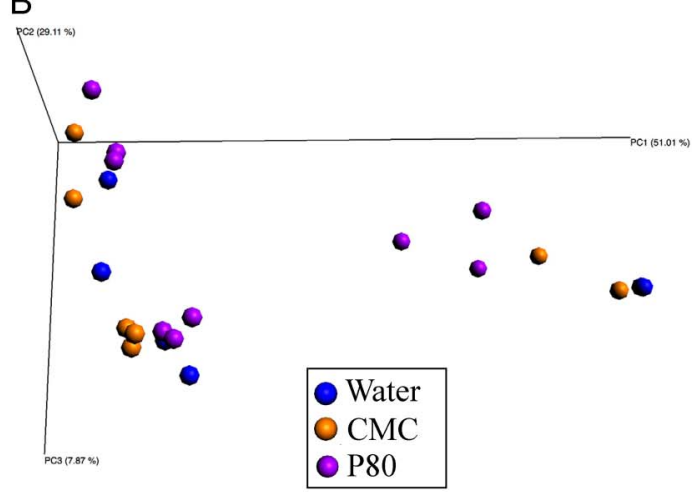

C
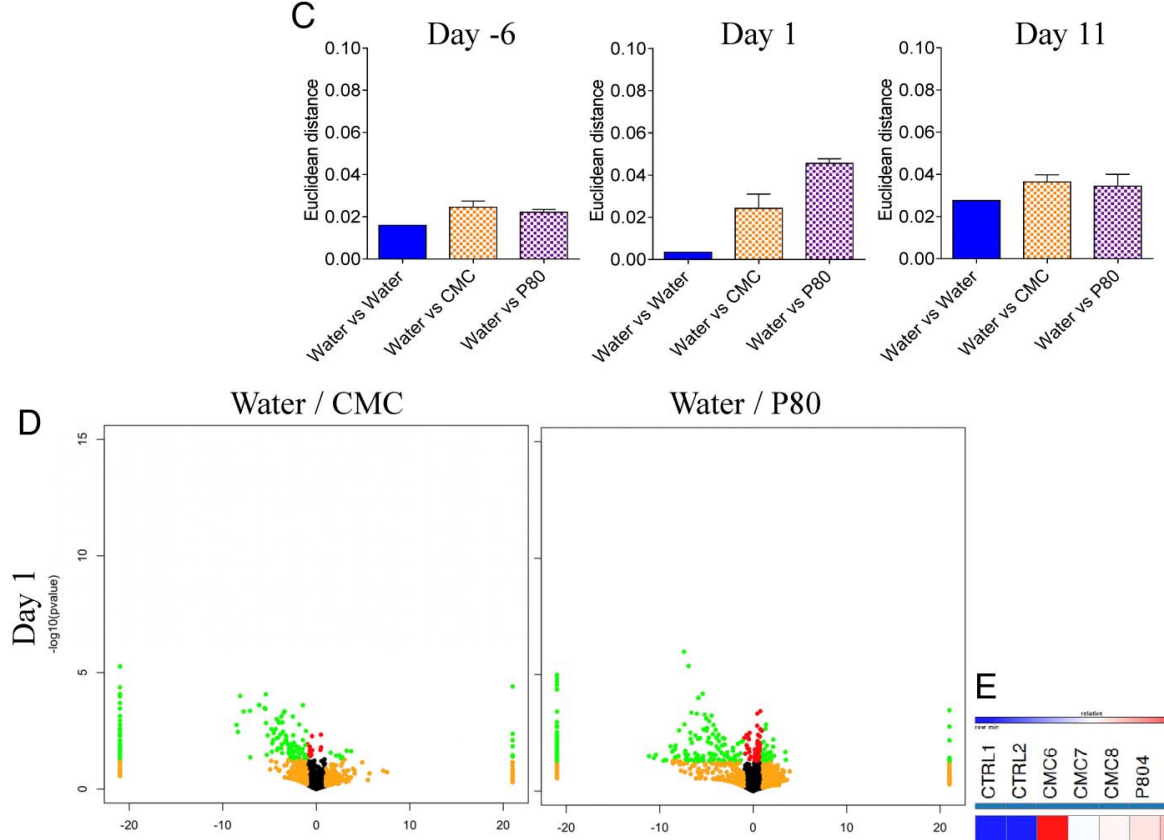

E
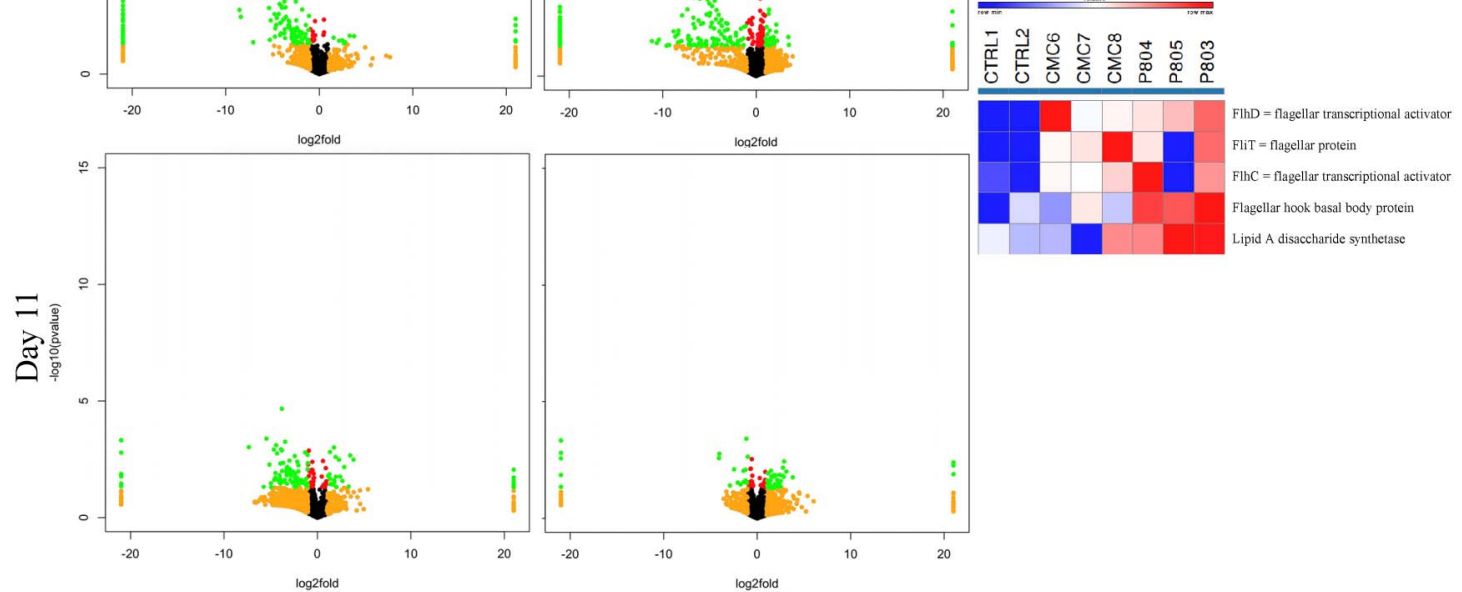

Figure 4 Both carboxymethylcellulose (CMC) and P80 alter microbial gene expression in the mucosal simulator of the human intestinal microbial ecosystem (M-SHIME) model. An eight-vessel M-SHIME system was set up and inoculated with fresh human faeces at day -7 . Following a 7-day stabilisation period, vessels were treated with either water $(\mathrm{N}=2), \mathrm{CMC} 1 \%(\mathrm{w} / \mathrm{v}, \mathrm{N}=3)$ or P80 $1 \%(\mathrm{v} / \mathrm{v}, \mathrm{N}=3)$ for 13 days. M-SHIME-associated luminal metatranscriptome was analysed by Illumina sequencing. (A and B) Following functional classification of the reads to Kyoto Encyclopedia of Genes and Genomes (KEGG) orthology classification at level 4 using MG-RAST, principal coordinates analysis of the Euclidean distance matrix was plotted at days $-6,1$ and 11. (C) Average of the Euclidean distance within group (water) and between group (water vs CMC and water vs P80) at days $-6,1$ and 11. (D) KEGG orthology (KO) at level 4 was visualised on a volcano plot. Up/left: water-treated versus CMC-treated at day 1; up/ right: water-treated versus P80-treated at day 1; bottom/left: water-treated versuS CMC-treated at day11; bottom/left: water-treated versus P80-treated at day11. For each KEGG identifier level 4, the difference in abundance between the two groups is indicated in log2 fold change on $x$-axis (with positive values corresponding to an increase in emulsifier-treated group compared with water-treated group, and negative values corresponding to a decrease in emulsifier-treated group compared with water-treated group), and significance between the two groups is indicated by $-\log 10 p$ value on the $y$-axis. Red dots correspond to KEGG identifiers with $p<0.05$ between emulsifier-treated and water-treated groups. Orange dots correspond to KEGG identifiers with at least a two-fold decreased or increased abundance in emulsifier-treated group compared with water-treated group. Green dots correspond to KEGG identifiers with at least a two-fold decreased or increased abundance in emulsifier-treated group compared with water-treated group and with $p<0.05$. (E) Heatmap representation using Gene-E of pathways related to flagella synthesis identified using KO, Clusters of Orthologous Groups of proteins and non-supervised orthologous groups. Data are the means $\pm \mathrm{SEM}(\mathrm{N}=2-3)$. 

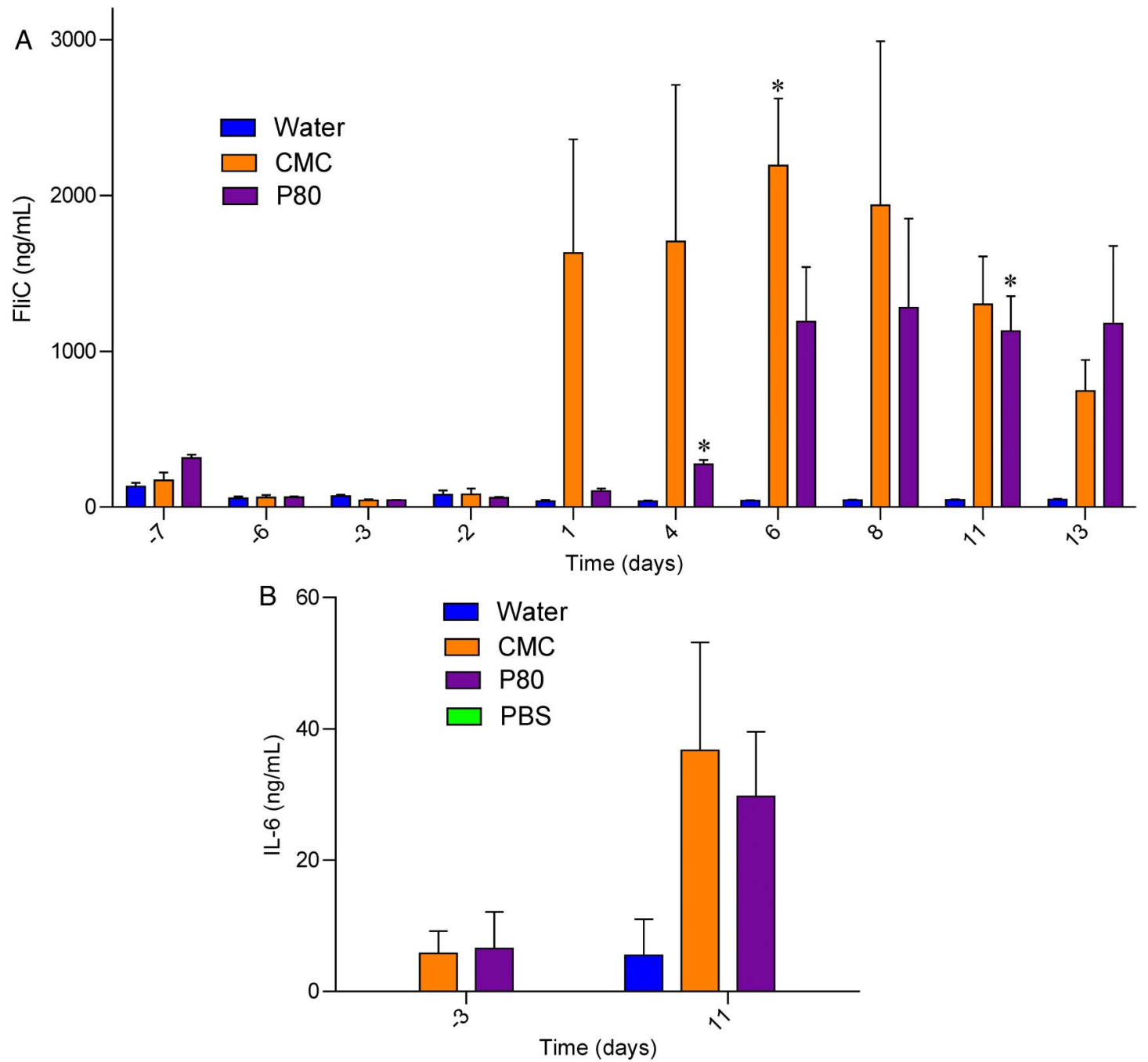

Figure 5 Carboxymethylcellulose (CMC) and P80 increase the proinflammatory potential of the mucosal simulator of the human intestinal microbial ecosystem (M-SHIME) microbiota. An eight-vessel M-SHIME system was set up and inoculated with fresh human faeces at day -7 . Following a 7-day stabilisation period, vessels were treated with either water $(\mathrm{N}=2), \mathrm{CMC} 1 \%(\mathrm{w} / \mathrm{v}, \mathrm{N}=3)$ or $\mathrm{P} 80 \mathrm{1} \%(\mathrm{v} / \mathrm{v}, \mathrm{N}=3)$ for 13 days. (A) Luminal M-SHIME suspension Flagellin (FliC) levels at days $-7,-6,-3,-2,1,4,6,8,11$ and 13. (B) Luminal M-SHIME suspension was injected intraperitoneally to $\mathrm{Rag}^{-1-}$ mice, and IL-6 was measured in the serum 2 hours post injection. Data are the means $\pm \mathrm{SEM}(\mathrm{N}=3)$. Each treatment group was compared with the control group (water-treated), and statistical significance was determined using Student's t-test.

was driven by increased $\alpha$-diversity (see online supplementary figure S2). All of the above analyses were also applied to M-SHIME mucus samples that yielded a very similar pattern of results (see online supplementary figure S3). Thus, P80, but not $\mathrm{CMC}$ exhibited a clear direct effect on the composition of a complex microbiota in the absence of a live host.

\section{Both CMC and P80 alter microbial gene expression in the M-SHIME model}

We next examined the extent to which CMC and P80 might influence microbial gene expression in the M-SHIME model. M-SHIME samples were assayed by RNA sequencing at days -6 , 6 and 11. PCoA analysis indicated that time in the M-SHIME model had a substantial effect on microbial gene expression, irrespective of emulsifier treatment in that all samples uniformly clustered based on the day of isolation (figure 4A). Nonetheless, M-SHIMEs treated with CMC or P80 following (day 1), but not preceding (day -6), emulsifier treatment exhibited clustering based on treatment as indicated visually and based on multidimensional (Euclidian) distance in PCoA coordinates (figure 4B, C). Display of relative gene expression data in volcano plots (figure 4D), scatter plots of relative expression between different time points and different conditions (see online supplementary figure $\mathrm{S} 4$ ) and summarising sequencing reads at different levels of the KEGG orthology classification (see online supplementary figure S5) indicated that neither CMC nor P80 broadly influenced gene expression but rather induced changes in a relative limited subset of genes. In accordance with this observation, assay of M-SHIME short-chain fatty acid and branchedchain fatty acid levels, which serve as a general read-out for gut microbiota metabolism and fermentation, did not differ appreciably in response to emulsifier treatment (see online supplementary figures S6 and S7). Importantly, one subset of genes whose transcripts were enriched in both CMC and P80 M-SHIMEs was of genes related to flagella expression (figure 4E). This result was reminiscent of results seen in emulsifier-treated mice, which displayed high level of faecal flagellin, which was hypothesised to promote low-grade inflammation via activation of TLR5 and/or NLRC4. ${ }^{8}$ Hence, we next assayed levels of bioactive flagellin in the M-SHIME model via 

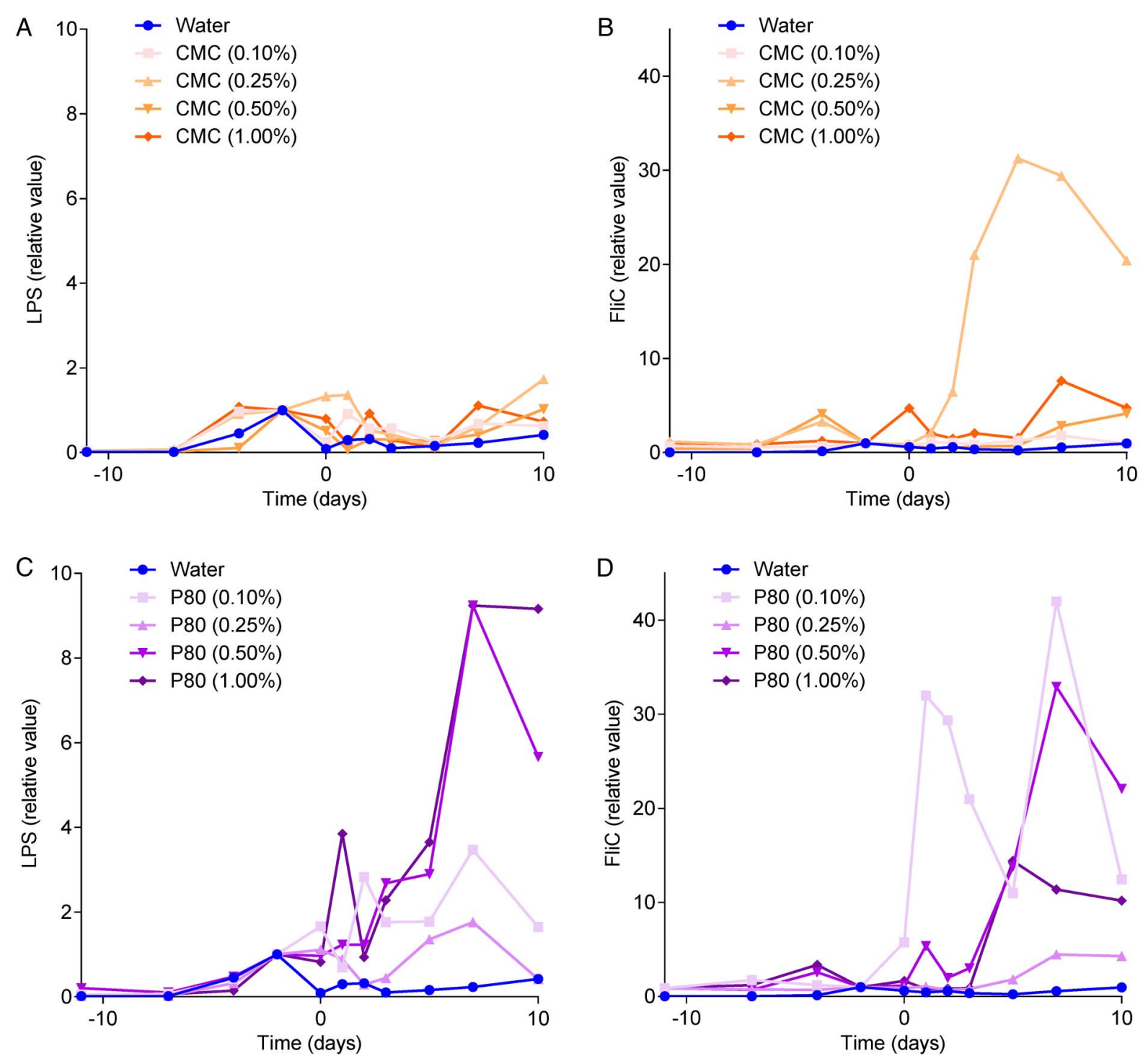

Figure 6 Carboxymethylcellulose (CMC) and P80 increase the proinflammatory potential of the mucosal simulator of the human intestinal microbial ecosystem (M-SHIME) microbiota. A nine-vessel M-SHIME system was set up and inoculated with fresh human faeces at day -11 . Following an 11-day stabilisation period, vessels were treated with either water, CMC $(0.10 \%, 0.25 \%, 0.50 \%$ or $1.00 \%)$ or P80 $(0.10 \%, 0.25 \%$, $0.50 \%$ or $1.00 \%$ ) for 10 days. (A and B) Luminal M-SHIME suspension lipopolysaccharide (LPS) (A) and Flagellin (FliC) (B) levels of CMC-treated M-SHIME at days $-10,-7,-4,-2,0,1,2,3,5,7$ and 10. (C and D) Luminal M-SHIME lipopolysaccharide (LPS) (C) and Flagellin (FliC) (D) levels of CMC-treated and P80-treated M-SHIME at days $-10,-7,-4,-2,0,1,2,3,5,7$ and 10 . Data are expressed as relative values with day -1 defined as $1(\mathrm{~N}=1)$.

the use of TLR5 reporter cells. CMC induced a robust increase in bioactive flagellin within 1 day of its administration that was presumably independent of alterations in microbial composition, which, as shown above, did not appreciably change in this model (figure 5A). In contrast, P80 induced a slower increase in M-SHIME flagellin levels that was evident 7 days following its administration, which is consistent with it resulting, at least in part, from an alteration in microbial composition (figure 5A), while no clear pattern was observed for another microbiotaderived proinflammatory molecule, lipopolysaccharide (see online supplementary figure S8). To more broadly examine how proinflammatory potential might be altered by CMC and P80 treatment, M-SHIME samples were injected into RAG $^{-/-}$ mice, which can make innate but not adaptive responses to bacteria and their products. As presented in figure 5B, suspensions from CMC-treated and P80-treated M-SHIME vessels induced a significantly higher IL-6 expression compared with water-treated M-SHIME vessels, further indicating that these emulsifying agents increased microbiota proinflammatory potential.

We next sought to investigate the concentration dependence of the effects of CMC and P80 in the M-SHIME model. M-SHIMEs were treated with $0.00 \%, 0.10 \%, 0.25 \%, 0.50 \%$ or $1.00 \%$ CMC or P80 (v/v and w/v, respectively). The relatively complex apparatus needed to maintain each M-SHIME unit as an independent ecosystem precluded us from running parallel replicates. Moreover, although these experiments used the same human donor used above to inoculate the M-SHIME vessels, the possibility that this donor's microbiome may have differed on the days the samples were provided for this and the abovedescribed experiments cannot be eliminated. Such caveats notwithstanding, we observed that all tested doses of P80 and all but the lowest dose of CMC elicited an increase in the levels of flagellin at multiple time points, although not displaying a clear dose-dependent linear relationship (figure 6A, C). P80 treatment also resulted in a clear increase in the levels of the TLR4-agonist 
A

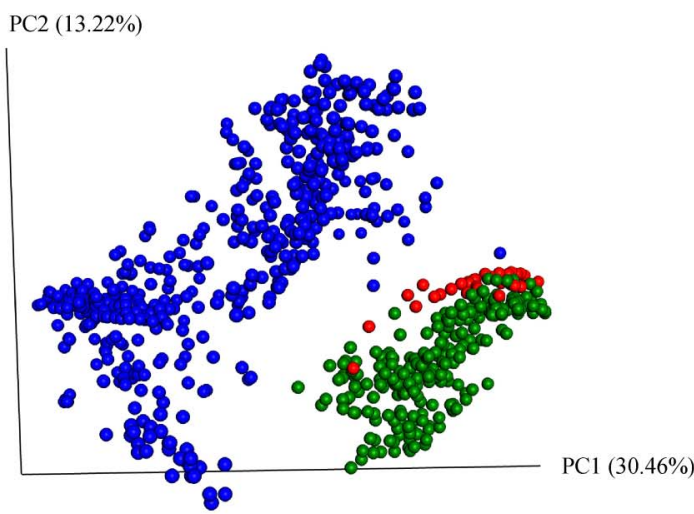

Conventional mice

- M-SHIME

M-SHIME transplant to germfree
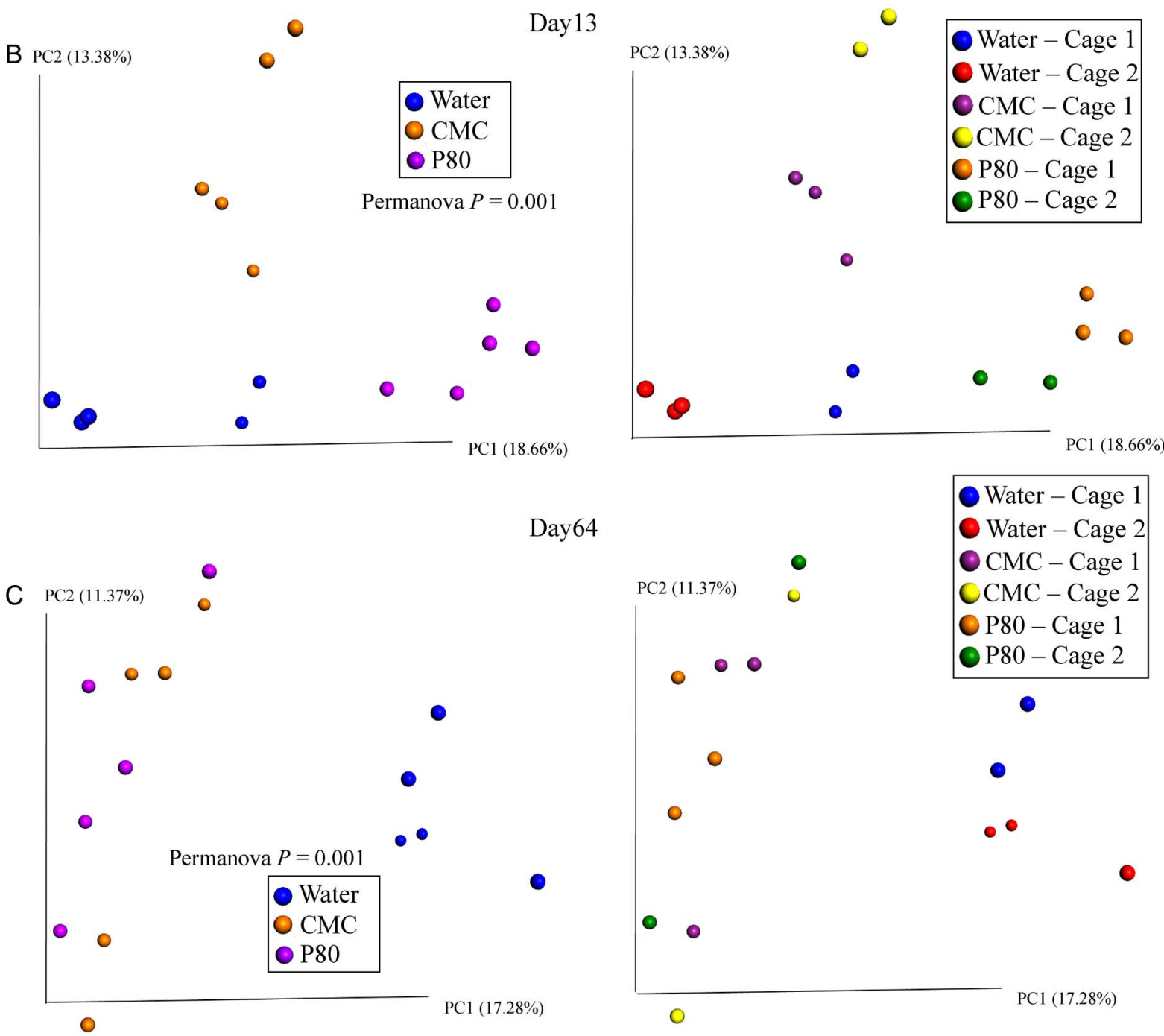

Day64

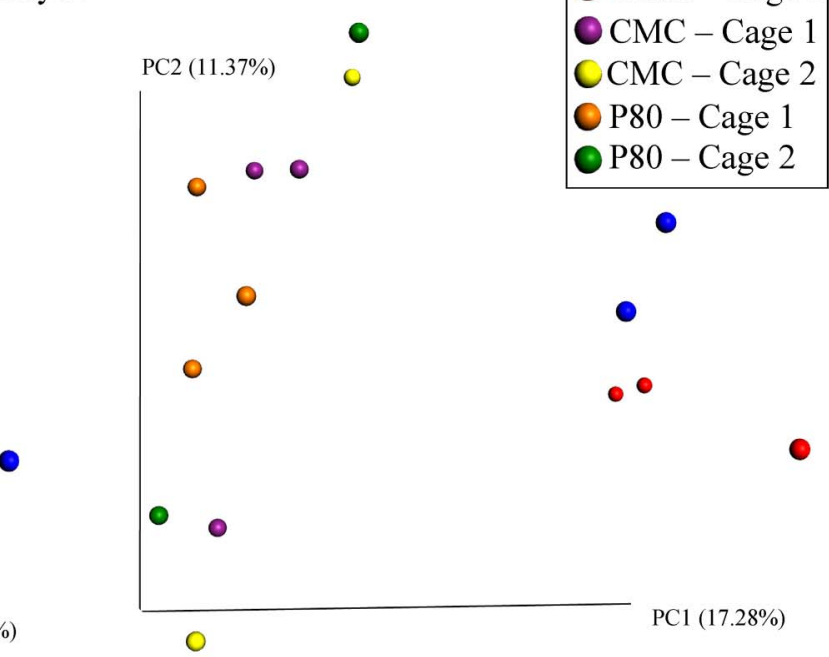

Figure 7 Altered microbiota composition in mice receiving carboxymethylcellulose (CMC)-treated and P80-treated mucosal simulator of the human intestinal microbial ecosystem (M-SHIME) suspensions. An eight-vessel M-SHIME system was set up and inoculated with fresh human faeces at day -7. Following a 7-day stabilisation period, vessels were treated with either water, CMC $1 \%$ or P80 1\% for 13 days. Germ-free C57BL/6 mice (3-4 weeks old) were removed from isolator and were orally administered with $200 \mu \mathrm{L}$ of luminal M-SHIME suspension (day 11 sample). Transplanted mice were then housed in isolated ventilated cages. For each condition (water-treated M-SHIME, CMC-treated M-SHIME, P80-treated M-SHIME), two cages containing two to three animals were used, with each cage receiving suspension from an independent M-SHIME vessel (for each condition, $\mathrm{N}=2$ vessels, $\mathrm{N}=2$ cages and $\mathrm{N}=5$ animals). Faecal microbiota composition was analysed using Illumina sequencing of the V4 region of 16S rRNA genes. (A) Microbiota composition was analysed from Georgia State University conventional mice, luminal M-SHIME suspension and germ-free mice colonised with luminal M-SHIME suspension using Illumina sequencing of the V4 region of 16S rRNA genes. (B and C) Principal coordinates analysis of the unweighted UniFrac distance matrix at days 13 (B) and 64 (C). Samples were coloured by treatment (left side) or by cage (right side) $(\mathrm{N}=5)$. For cluster analysis on principal coordinate plots, categories were compared, and statistical significance of clustering was determined using the Permanova method. 
LPS, especially at higher doses (figure 6B, D). Together, these results indicated that $\mathrm{CMC}$ and $\mathrm{P} 80$ can act directly on a complex microbiota in a manner that might increase its potential to promote inflammation in the host.

\section{CMC-treated and P80-treated M-SHIME suspensions promote low-grade inflammation-associated phenotypes in mice}

We next examined the extent to which emulsifier-induced alterations of M-SHIME microbiotas might have the potential to impact a host by transferring them to GF mice. The $16 \mathrm{~S}$ sequencing analysis of faeces from M-SHIME recipient mice revealed that, relative to a range of actual M-SHIME samples and faecal samples from conventional mice (from ongoing microbiome-related studies), M-SHIME recipient mice had microbiomes that, although distinct from both aforementioned groups, were much closer in overall composition to M-SHIME microbiomes, indicating that our approach preserved much of the basic nature of the M-SHIME microbiota many weeks following transplant (figure 7A). Moreover, at both times assayed, microbiota composition of M-SHIME recipient mice clearly clustered based on M-SHIME treatment, irrespective of cage clustering (figure $7 \mathrm{~B}, \mathrm{C}$ ), including alteration of $\alpha$-diversity in mice receiving emulsifier-treated M-SHIME suspensions compared with those receiving water-treated M-SHIMEs (see online supplementary figure S9A, B). Such altered microbiota composition was characterised by an increase in inflammation-associated bacteria, such as Proteobacteria and Enterobacteriacae, and decreased levels of health-associated bacteria, such as Bacteroidaceae, in mice receiving emulsifier-treated M-SHIME microbiotas (see online supplementary figure S9C-F). Predicted metagenomes based on this $16 \mathrm{~S}$ composition, using PICRUSt (phylogenetic investigation of communities by reconstruction of unobserved states), ${ }^{26}$ indicated that the mice receiving emulsifier-treated M-SHIME-derived microbiotas harboured an altered metagenome compared with those receiving watertreated M-SHIMEs, characterised by an enrichment of genes related to flagella/motility (see online supplementary figures S10 and S11). Accordingly, at both time points assayed, recipients of CMC-treated and P80-treated M-SHIMEs displayed higher levels of bioactive flagellin and LPS (figure $8 \mathrm{~A}-\mathrm{D}$ ). Thus, some aspects of CMC-induced and P80-induced M-SHIME microbiota alterations persisted for a prolonged period in vivo following transfer to GF mice.

We next analysed the extent to which such conventionalisation of GF mice with emulsifier-altered microbiotas might correlate with altered metabolic and/or intestinal phenotypes. Conventionalisation of GF mice is known to restore a more normal gut morphology. ${ }^{27} 28$ Accordingly, relative to GF mice, those colonised with M-SHIME water-treated suspension exhibited an increase in intestinal mass, which was not associated with any indications of metabolic syndrome (see online supplementary figure S12). Conventionalisation of GF mice is also known to be followed by weight gain. Accordingly, although we did not track weight of GF mice (as it risks breaching the GF state), we observed that all groups of GF mice receiving M-SHIMEs more than doubled their body mass within 2 weeks of receiving M-SHIMEs. The extent of such weight gain did not differ between mice receiving water-treated and emulsifier-treated M-SHIMEs (figure $8 \mathrm{E}$ ), but recipients of both CMC-treated and P80-treated M-SHIMEs exhibited higher levels of fasting blood glucose following a 15 hours or 5 hours fasting period (figure $8 \mathrm{~F}, \mathrm{G}$ ), often used for human and mouse studies, respectively, thus suggesting that emulsifier-treated microbiotas might promote this central feature of metabolic syndrome. This notion was further supported by post-euthanasia assay of these animals, which indicated that mice receiving emulsifier-treated M-SHIMES displayed a range of features that are associated with low-grade inflammation and metabolic syndrome. Specifically, relative to mice receiving water-treated M-SHIME suspensions, recipients of CMC-treated and P80-treated M-SHIMEs displayed shortened thickened colons, mild splenomegaly, elevated faecal Lcn2 levels and increased adiposity, as indicated by epididymal fat pad mass (figure $8 \mathrm{H}-\mathrm{N}$ ). Importantly, such low-grade inflammatory phenotypes correlated with microbiota encroachment by the emulsifier-treated M-SHIME microbiotas (figure 9).

Some alterations in microbiota are known to predispose to weight gain upon exposure to an obesogenic HFD. ${ }^{29-31}$ Hence, we analysed the extent to which emulsifier-treated M-SHIME suspension inoculation would impact GF recipient mice upon feeding the colonised animals HFD. We observed that upon HFD feeding, mice receiving emulsifier-treated M-SHIME suspensions gained more weight than those receiving water-treated M-SHIMEs. Such increased weight gain correlated with elevated levels of fasting blood glucose (see online supplementary figure $\mathrm{S} 13 \mathrm{C}$ ) and inflammation, such as a colon shortening (see online supplementary figure S13F) and splenomegaly (see online supplementary figure $\mathrm{S} 13 \mathrm{H})$. Together, these results suggest that direct effects of CMC and P80 on the microbiota play a role in driving the inflammometabolic phenotypes induced by these compounds in vivo.

\section{DISCUSSION}

The gut microbiota is a complex ecosystem that wields great influence over the health of its host. ${ }^{32}$ Conversely, mammalian hosts use a broad array of mechanisms to shape the microbiota in a way that maximises its benefit and minimises its potential to harm. ${ }^{33}$ That, at least among healthy persons, intraindividual variance in microbiota composition (ie, repeat sampling of individual subjects) is much smaller than that seen when comparing different individuals ${ }^{33-35}$ indicates that the ability of host and microbiota to influence each other typically results in a relatively stable equilibrium within specific hosts. Yet, some exogenous factors, for example, pathogens or antibiotics, can dramatically and lastingly disturb this equilibrium in a manner that promotes persistent disease. ${ }^{36}$ Moreover, even factors that cause relatively modest disturbances of the host-microbiota relationship have been associated with chronic low-grade inflammatory diseases, including metabolic syndrome. ${ }^{36}$ The genetics of the host is surely a pivotal determinant of the resiliency of host-microbiota equilibrium following exposure to perturbants. For example, pathobionts that are readily cleared by WT mice without evidence of an associated phenotype can trigger lasting colitis in mice with an array of genetic abnormalities. ${ }^{37}$ Such colitis is associated with, and promoted by, the altered microbiota, which can remain lastingly disturbed long after the pathobiont has been cleared. Analogously, the dietary emulsifiers CMC and P80 induce a moderate disturbance of microbiota in WT mice that associates with low-grade inflammation and metabolic disease, but promotes robust colitis, associated with a more severe alteration of gut microbiota composition, in mice lacking IL-10 or TLR5. ${ }^{8}$

On the one hand, the ability of the host and microbiota to influence each other precludes the use of microbiota-free hosts (ie, GF mice) or host-free microbiotas (eg, M-SHIMEs) in fully defining how CMC or P80 (or other substances) ultimately impacts the host or microbiota. But on the other hand, such bi-directional host-microbiota interactions dictate that use of 

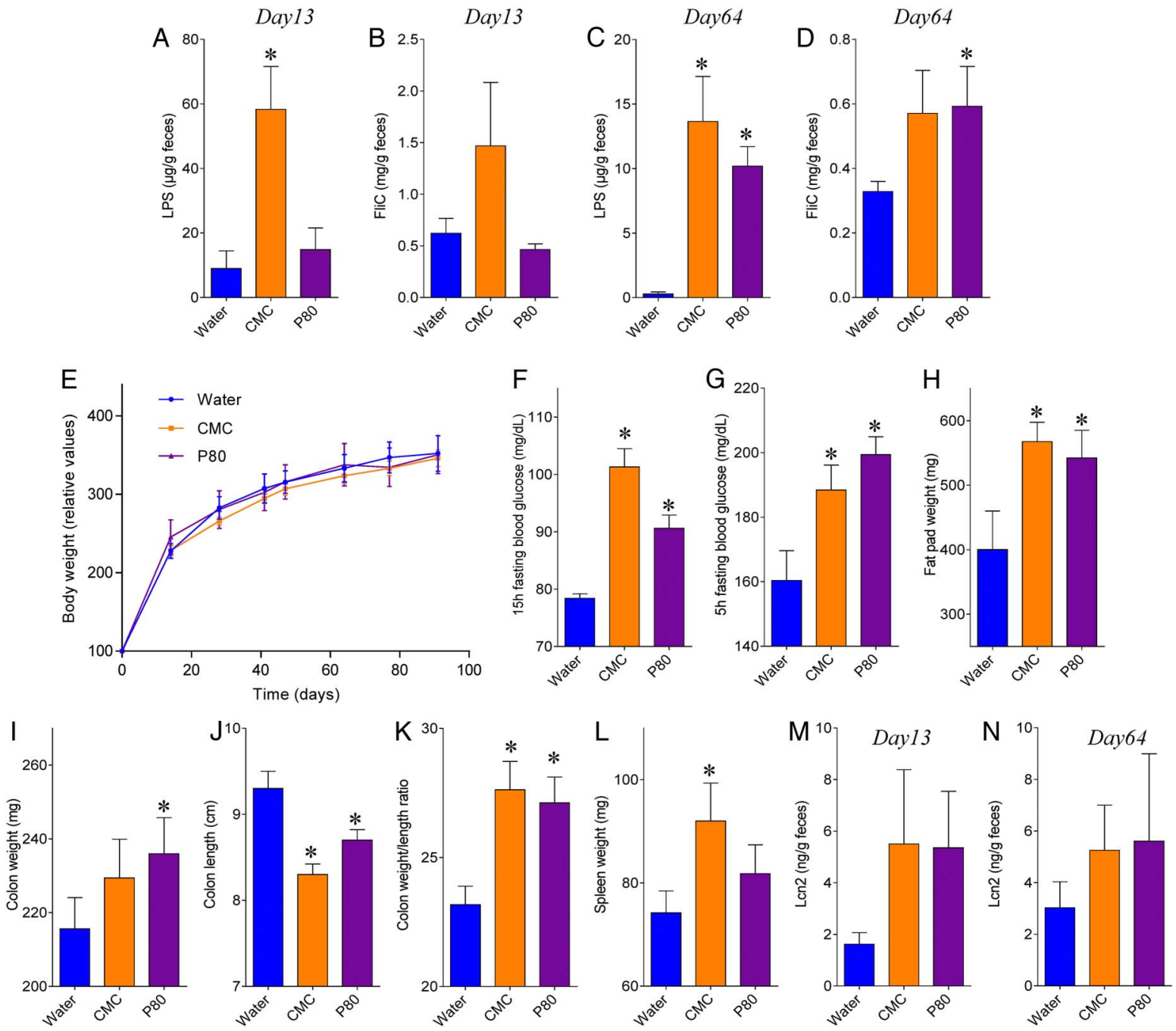

Figure 8 Carboxymethylcellulose (CMC)-treated and P80-treated M-SHIME suspensions promote low-grade inflammation when transplanted to germ-free recipient mice. An eight-vessel mucosal simulator of the human intestinal microbial ecosystem (M-SHIME) system was set up and inoculated with fresh human faeces at day -7 . Following a 7-day stabilisation period, vessels were treated with either water, CMC $1 \%$ or P80 $1 \%$ for 13 days. Germ-free C57BL/6 mice (3-4 weeks old) were removed from the isolator and were orally administered with $200 \mu \mathrm{L}$ of luminal M-SHIME suspension (day 11 sample). Transplanted mice were then housed in isolated ventilated cages. For each condition (water-treated, CMC-treated and P80-treated M-SHIME), two cages containing two to three animals were used, with each cage receiving suspension from an independent M-SHIME vessel (for each condition, $\mathrm{N}=2$ vessels, $\mathrm{N}=2$ cages and $\mathrm{N}=5$ animals). (A and C) lipopolysaccharide (LPS) and (B and D) Flagellin (FliC) levels at days 13 (A and B) and 64 (C and D). (E) Body weight over time, (F) 15 hours fasting blood glucose concentration, (G) 5 hours fasting blood glucose concentration, $(\mathrm{H})$ fat pad weight, (I) colon weight, $(\mathrm{J})$ colon length, $(\mathrm{K})$ colon weight/length ratio, (L) spleen weight and (M and N) faecal Lcn2 levels at days $13(\mathrm{M})$ and $64(\mathrm{~N})$. Data are the means $\pm \mathrm{SEM}(\mathrm{N}=5)$. Each treatment group was compared with the control group (water-treated), and statistical significance was determined using Student's t-test.

these methodologies may be the only means to define direct mechanisms by which an exogenous agent perturbs the hostmicrobiota relationship. Indeed, we reasoned that the detergentlike properties of CMC and P80 might allow them to alter the physicochemical properties of the mucus layer that protects the gut from the microbiota. ${ }^{8} 9{ }^{12}$ However, that we have not observed such effects by these compounds in GF mice prompted us to consider that the microbiota itself may be a direct target of CMC or P80. That CMC and P80 altered gene expression and composition in the M-SHIME model in a manner somewhat reminiscent of their effects in mice supports the notion that, in vivo, the bioactions of $\mathrm{CMC}$ and P80 effects involve direct effects on the microbiota. Conversely, we speculate that CMC not inducing a clear change in microbial composition in the M-SHIME model, but doing so in mice, may reflect that the altered composition in vivo is driven, in part, by the host response to the $\mathrm{CMC}$-induced changes in microbial gene expression. Indeed, an increase in flagella-related gene expression, which CMC induced in the M-SHIME model, can be readily envisaged, in vivo, to increase activation of TLR5 and/or NLRC4 by both providing higher intestinal concentrations of flagellin and greater ability of motile bacteria to penetrate the mucus layer, increasing the likelihood that flagellin released by bacteria would reach host receptors capable of recognising it. Activation of these receptors would increase host expression of a range of antimicrobial peptides that would have the potential to shift microbiota composition.

That transfer of CMC-treated and P80-treated M-SHIMEs to GF mice transferred features of low-grade inflammation/metabolic syndrome supports the notion that the direct actions of these compounds observed in the M-SHIME model play a central role in mediating their detrimental effects on the host. 

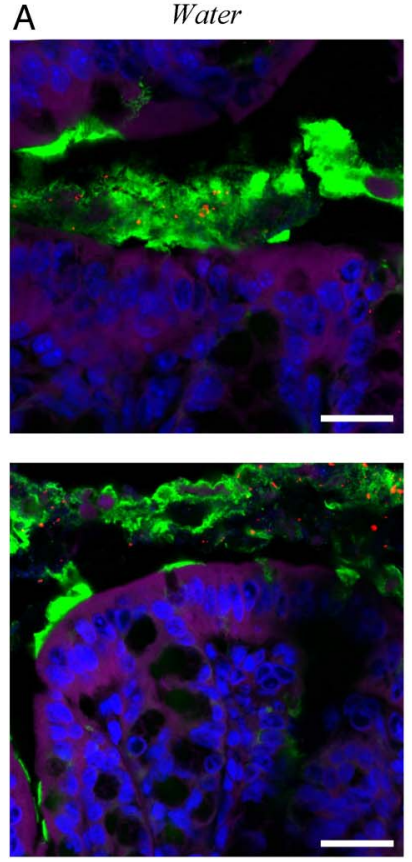

$C M C$
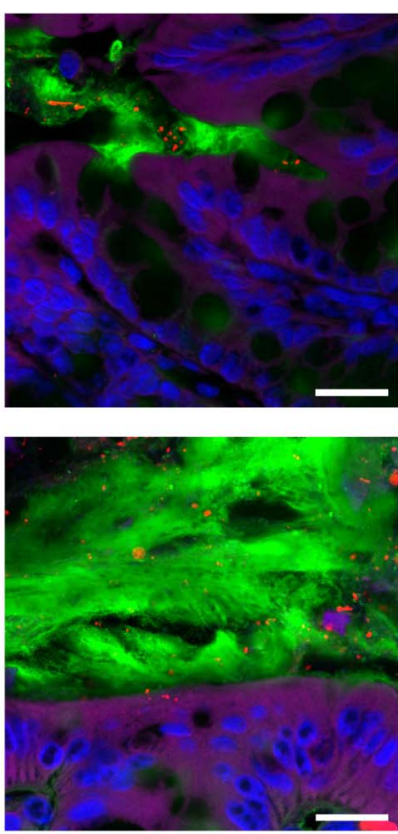

P80
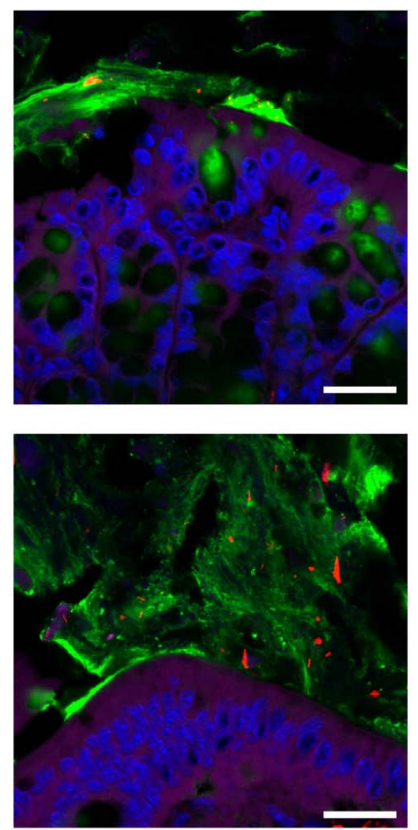

$\mathrm{B}$

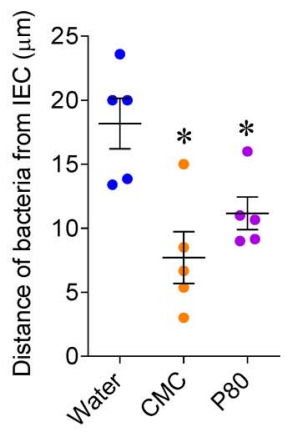

Figure 9 Carboxymethylcellulose (CMC)-treated and P80-treated mucosal simulator of the human intestinal microbial ecosystem (M-SHIME) suspensions promote microbiota encroachment when transplanted to germ-free recipient mice. An eight-vessel M-SHIME system was set up and inoculated with fresh human faeces at day -7. Following a 7-day stabilisation period, vessels were treated with either water, CMC $1 \%$ or P80 $1 \%$ for 13 days. Germ-free C57BL/6 mice (3-4 weeks old) were removed from the isolator and were orally administered with $200 \mu \mathrm{L}$ of luminal M-SHIME suspension (day 11 sample). Transplanted mice were then housed in isolated ventilated cages, Isocages (Techniplast). (A) Confocal microscopy analysis of microbiota localisation: Muc2 (green), actin (purple), bacteria (red) and DNA (blue). Bar=20 $\mu$ m. (B) Distances of the closest bacteria to intestinal epithelial cells (IEC) per condition over three high-powered fields per mouse. Data are the means $\pm \mathrm{SEM}(\mathrm{N}=5)$. Each treatment group was compared with the control group (water-treated), and statistical significance was determined using Student's t-test.

We hypothesise that the key feature of emulsifier-treated M-SHIME microbiotas that drove their ability to promote inflammation following transplant was their greater ability to encroach upon host cells whereupon their products (eg, LPS and flagellin) would induce proinflammatory gene expression, including production of cytokines that can cross-desensitise metabolic receptor signalling. ${ }^{12} 38$

Differences in microbiota and host parameters between GF mice receiving water-treated and emulsifier-treated M-SHIMEs persisted many weeks following M-SHIMEs transplant. Yet, given that any residual CMC or P80 present during the transplant would be quickly washed out upon transfer to GF mice, it might have been reasonable to presume that the microbiota would quickly revert to its untreated state. We speculate such persistence reflects that the initial host response to the altered M-SHIMEs maintains and/or enhances alterations in microbiota gene expression, and leads to further alterations in composition. The lack of beneficial microbiota reversion in mice receiving CMC-treated and P80-treated M-SHIMEs might also be a consequence of our approach not permitting a normal mouse microbiota to outcompete a disturbed human microbiome, which may be more difficult for the mouse gut to corral. Regardless, these observations highlight the importance of early host-microbiota interactions in having a lasting impact on the host-microbiota relationship and, consequently, host health.

In conclusion, while we do not believe the M-SHIME or any host-free microbiota can fully define how an agent would impact the microbiota within its host, our results demonstrate that this system can be a very useful tool to explore underlying mechanisms. The M-SHIME model has enabled us to conclude that our observation that the emulsifiers CMC and P80, which have been previously observed to impact the mouse microbiome, also impact human microbiomes, at least in this ex vivo model. This observation should enable us to better design human clinical trial on dietary emulsifiers, which we hope to initiate soon. Furthermore, our results described herein strongly suggest that the microbiota is one direct important target of the food additives CMC and P80. We envision that this model may allow further mechanistic investigation into how these compounds directly alter the microbiota. Moreover, our results suggest that such models may be a useful means to develop food additives and medicine vehicles that maintain their intended functions without negatively impacting the microbiome.

Acknowledgements The authors thank Dr Amanda Ramer-Tait for the sequences of ASF members specific primers. ${ }^{39}$

Contributors Conception and design: BC, TVdW and ATG. Development of methodology: BC, TVdW and ATG. Acquisition of data: BC and JDB. Analysis and interpretation of data: BC, TVdW, JDB, MM and ATG. Writing, review and/or revision of the manuscript: BC and ATG. Study supervision: ATG.

Funding This work was supported by NIH grant DK099071 (ATG) and DK083890 (ATG). BC is a recipient of the Career Development Award from the Crohn's and Colitis Foundation of America (CCFA). TVdW is supported by UGent grant BOF2017-GOA-032. MM is supported by a postdoctoral mandate from Research Foundation Flanders (FWO).

\section{Competing interests None declared.}

Provenance and peer review Not commissioned; externally peer reviewed.

Data sharing statement Metatranscriptomic: unprocessed sequencing data are deposited at MG-RAST http://metagenomics.anl.gov/linkin.cgi?project=mgp16059, samples mgs411584, mgs411587, mgs411590, mgs411593, mgs411596, mgs411599, mgs411602, mgs411605, mgs411608, mgs411611, mgs411614, mgs411617, mgs411620, mgs411623, mgs411626, mgs411629, mgs411632, mgs411635, mgs411638, mgs411641, mgs411644, mgs411647, mgs411650, mgs411653. 16S sequencing: unprocessed sequencing data are deposited in the European Nucleotide Archive under accession numbers PRJEB19279 (M-SHIME suspension) and PRJEB19272 (transplant of M-SHIME suspension to germ-free recipient mice). 


\section{REFERENCES}

1 Dalal SR, Chang EB. The microbial basis of inflammatory bowel diseases. J Clin Invest 2014;124:4190-6.

2 Lupp C, Robertson ML, Wickham ME, et al. Host-mediated inflammation disrupts the intestinal microbiota and promotes the overgrowth of Enterobacteriaceae. Cell Host Microbe 2007;2:204.

3 Spees AM, Wangdi T, Lopez CA, et al. Streptomycin-induced inflammation enhances Escherichia coli gut colonization through nitrate respiration. mBio 2013;4:e00430-13.

4 Faber $F$, Bäumler AJ. The impact of intestinal inflammation on the nutritional environment of the gut microbiota. Immunol Lett 2014;162:48-53.

5 Winter SE, Thiennimitr $\mathrm{P}$, Winter MG, et al. Gut inflammation provides a respiratory electron acceptor for Salmonella. Nature 2010;467:426-9.

6 Devkota S, Wang Y, Musch MW, et al. Dietary-fat-induced taurocholic acid promotes pathobiont expansion and colitis in II10-/- mice. Nature 2012:487:104-8.

7 Devkota S, Chang EB. Diet-induced expansion of pathobionts in experimental colitis: implications for tailored therapies. Gut Microbes 2013;4:172-4.

8 Chassaing B, Koren 0, Goodrich JK, et al. Dietary emulsifiers impact the mouse gut microbiota promoting colitis and metabolic syndrome. Nature 2015;519:92-6.

9 Roberts CL, Keita AV, Duncan SH, et al. Translocation of Crohn's disease Escherichia coli across M-cells: contrasting effects of soluble plant fibres and emulsifiers. Gut 2010;59:1331-9.

10 Roberts CL, Rushworth SL, Richman E, et al. Hypothesis: Increased consumption of emulsifiers as an explanation for the rising incidence of Crohn's disease. J Crohns Colitis 2013;7:338-41.

11 Swidsinski A, Ung V, Sydora BC, et al. Bacterial overgrowth and inflammation of small intestine after carboxymethylcellulose ingestion in genetically susceptible mice. Inflamm Bowel Dis 2009;15:359-64.

12 Chassaing B, Gewirtz AT. Has provoking microbiota aggression driven the obesity epidemic? Bioessays 2016;38:122-8.

13 Van den Abbeele $P$, Roos $S$, Eeckhaut $V$, et al. Incorporating a mucosal environment in a dynamic gut model results in a more representative colonization by lactobacilli. Microb Biotechnol 2012;5:106-15.

14 Van den Abbeele P, Belzer C, Goossens M, et al. Butyrate-producing Clostridium cluster XIVa species specifically colonize mucins in an in vitro gut model. ISME J 2013;7:949-61.

15 Geirnaert A, Wang J, Tinck $M$, et al. Interindividual differences in response to treatment with butyrate-producing Butyricicoccus pullicaecorum 25-3T studied in an in vitro gut model. FEMS Microbiol Ecol 2015;91:fiv054.

16 Marzorati M, Vilchez-Vargas R, Bussche JV, et al. High-fiber and high-protein diets shape different gut microbial communities, which ecologically behave similarly under stress conditions, as shown in a gastrointestinal simulator. Mol Nutr Food Res 2017:61.

17 Metcalf AM, Phillips SF, Zinsmeister AR, et al. Simplified assessment of segmental colonic transit. Gastroenterology 1987;92:40-7.

18 Nugent SG, Kumar D, Rampton DS, et al. Intestinal luminal pH in inflammatory bowel disease: possible determinants and implications for therapy with aminosalicylates and other drugs. Gut 2001;48:571-7.

19 Meyer F, Paarmann D, D'Souza M, et al. The metagenomics RAST server-a public resource for the automatic phylogenetic and functional analysis of metagenomes. BMC Bioinformatics 2008;9:386.
20 Kanehisa M, Sato Y, Kawashima M, et al. KEGG as a reference resource for gene and protein annotation. Nucleic Acids Res 2016;44:D457-62.

21 Kanehisa M, Goto S. KEGG: kyoto encyclopedia of genes and genomes. Nucleic Acids Res 2000;28:27-30.

22 Tatusov RL, Galperin MY, Natale DA, et al. The COG database: a tool for genome-scale analysis of protein functions and evolution. Nucleic Acids Res 2000;28:33-6.

23 Huerta-Cepas J, Szklarczyk D, Forslund K, et al. eggNOG 4.5: a hierarchical orthology framework with improved functional annotations for eukaryotic, prokaryotic and viral sequences. Nucleic Acids Res 2016;44:D286-93.

$24 \mathrm{http}: / /$ www.broadinstitute.org/cancer/software/GENE-E/index.html

25 Hecht G, Bar-Nathan C, Milite G, et al. A simple cage-autonomous method for the maintenance of the barrier status of germ-free mice during experimentation. Lab Anim 2014;48:292-7.

26 Langille MG, Zaneveld J, Caporaso JG, et al. Predictive functional profiling of microbial communities using $16 \mathrm{~S}$ rRNA marker gene sequences. Nat Biotechnol 2013;31:814-21.

27 Musso G, Gambino R, Cassader M. Obesity, diabetes, and gut microbiota: the hygiene hypothesis expanded? Diabetes care 2010;33:2277-84.

28 Bäackhed $\mathrm{F}$, Ding $\mathrm{H}$, Wang $\mathrm{T}$, et al. The gut microbiota as an environmental factor that regulates fat storage. Proc Natl Acad Sci USA 2004;101: 15718-23.

29 Fujisaka S, Ussar S, Clish C, et al. Antibiotic effects on gut microbiota and metabolism are host dependent. J Clin Invest 2016;126:4430-43.

30 Bäckhed F, Manchester JK, Semenkovich CF, et al. Mechanisms underlying the resistance to diet-induced obesity in germ-free mice. Proc Natl Acad Sci USA 2007; 104:979-84.

31 Turnbaugh PJ, Ley RE, Mahowald MA, et al. An obesity-associated gut microbiome with increased capacity for energy harvest. Nature 2006;444:1027-31.

32 Rooks MG, Garrett WS. Gut microbiota, metabolites and host immunity. Nat Rev Immunol 2016;16:341-52.

33 Lloyd-Price J, Abu-Ali G, Huttenhower C. The healthy human microbiome. Genome Med 2016:8:51.

34 Hisada T, Endoh K, Kuriki K. Inter- and intra-individual variations in seasonal and daily stabilities of the human gut microbiota in Japanese. Arch Microbiol 2015; 197:919-34.

35 Lazarevic V, Whiteson K, Hernandez D, et al. Study of inter- and intraindividual variations in the salivary microbiota. BMC Genomics 2010; 11:523.

36 Cox LM, Yamanishi S, Sohn J, et al. Altering the intestinal microbiota during a critical developmental window has lasting metabolic consequences. Cell 2014;158:705-21.

37 Chassaing B, Koren O, Carvalho FA, et al. AIEC pathobiont instigates chronic colitis in susceptible hosts by altering microbiota composition. Gut 2014;63:1069-80.

38 Gregor MF, Hotamisligil GS. Inflammatory mechanisms in obesity. Annu Rev Immunol 2011:29:415-45.

39 Gomes-Neta JC, Mantz S, Held K, et al. A new real-time PCR assay for accurate quantification of the individual members of the Altered Schaedler Flora microbiota in gnotobiotic mice. J Microbiol Methods 2017;135:52-62. 